\title{
Modules of bounded length in Auslander-Reiten components
}

\author{
By \\ Eugenia Marmolejo and Claus Michael Ringel
}

Let $A$ be an artin algebra, and $\Gamma$ a component of the Auslander-Reiten quiver of $A$. It has been asked [12] whether the number of isomorphism classes of indecomposable $A$-modules in $\Gamma$ of fixed length is finite. This question is answered affirmatively for certain types of regular components. More generally, we consider regular components of full additive subcategories with sink maps in a length category.

Recall that a length category is an abelian category where every object $X$ has a finite composition series; the length of such a composition series will be called the length of $X$ and denoted by $|X|$. We denote by $[X]$ the isomorphism class of the object $X$, and we also write $|[X]|$ instead of $|X|$. Let $\mathscr{A}$ be a length category, and $\mathscr{K}$ a full additive subcategory. We consider $\mathscr{K}$ as a Krull-Schmidt category with short exact sequences, the short exact sequences being those in $\mathscr{A}$ which belong to $\mathscr{K}$. Thus, the Auslander-Reiten quiver $\Gamma(\mathscr{K})$ of $\mathscr{K}$ is defined (see [11]), but we consider $\Gamma(\mathscr{K})$ as a valued translation quiver, see [5], and we denote by $\tau_{\mathscr{K}}$ the Auslander-Reiten translation in $\mathscr{K}$. An (Auslander-Reiten) component $\mathscr{C}$ of $\mathscr{K}$ is, by definition, the full additive subcategory generated by the indecomposable objects $X$ in $\mathscr{K}$ whose isomorphism classes belong to a fixed component $\Gamma^{\prime}$ of $\Gamma(\mathscr{K})$; of course, $\Gamma^{\prime}=\Gamma(\mathscr{C})$. A component $\mathscr{C}$ of $\mathscr{K}$ is said to be regular provided $\Gamma(\mathscr{C})$ is a stable translation quiver. (Note that a component $\mathscr{C}$ of $\mathscr{K}$ is regular if and only if the following condition is satisfied: if $X$ is indecomposable in $\mathscr{C}$, then there exists a source map $f$ and a sink map $g$ for $X$ in $\mathscr{K}$, and there are short exact sequences $\left(f, f^{\prime}\right)$ and $\left(g^{\prime}, g\right)$ which lie inside $\mathscr{C}$.) Let $\mathscr{C}$ be a regular component of $\mathscr{K}$. We assume in addition that there are indecomposable objects $C_{1}, C_{2}$ in $\mathscr{C}$ and $X_{1}, X_{2}$ in $\mathscr{K} \backslash \mathscr{C}$, with Hom $\left(X_{1}, C_{1}\right) \neq 0$ and $\operatorname{Hom}\left(C_{2}, X_{2}\right) \neq 0$. Finally, assume there is a constant $d$ such that for any indecomposable object $X$ of $\mathscr{C}$, we have $\left|\tau_{\mathscr{H}} X\right| \leqq d|X|$. These conditions are obviously satisfied in the case that $\mathscr{B}$ is a stable component of $\mathscr{A}=\mathscr{K}=A$-mod. (For $d$, we may take $\left(\operatorname{dim}_{k} A\right)^{2}$, see [10]). They are also satisfied in the following situation: Let ${ }_{A} T$ be a tilting module, $\mathscr{K}=\mathscr{G}\left({ }_{A} T\right)$ the full subcategory of all $A$-modules generated by ${ }_{A} T$, and $\mathscr{A}=A$-mod. (Here, take for $X_{1}$ a suitable dirct summand of ${ }_{A} T$, for $X_{2}$ a suitable indecomposable injective $A$-module; also if $\tau_{\mathscr{K}} X$ exists, it can be embedded into $\tau X$, see [7], thus, again let $d=\left(\operatorname{dim}_{k} A\right)^{2}$.)

A valued quiver $\Delta=\left(\Delta_{0}, \Delta_{1}, d, d^{\prime}\right)$ is given by a quiver $\left(\Delta_{0}, \Delta_{1}\right)$ without multiple arrows, and two functions $d, d^{\prime}$ defined on $\Delta_{1}$ with values in the set $\mathbb{N}_{1}$ of positive integers, if $\alpha: a \rightarrow b$ is an arrow, we write $d_{a b}$ instead of $d(\alpha)$ and $d_{a b}^{\prime}$ instead of $d^{\prime}(\alpha)$. A valued translation quiver $I^{T}=\left(\Gamma_{0}, \Gamma_{1}, d, d^{\prime}, \tau\right)$ is given by a valued quiver $\left(\Gamma_{0}, \Gamma_{1}, d, d^{\prime}\right)$ and a 
translation quiver $\left(\Gamma_{0}, \Gamma_{1}, \tau\right)$ such that for any non-projective vertex $z$ and any arrow $y \rightarrow z$, we have $d_{\tau z, y}=d_{y z}^{\prime}$ and $d_{\tau z, y}^{\prime}=d_{y z}$. Given a valued quiver $A$, following Riedtmann $[9,5]$ we define a valued translation quiver $\mathbb{Z} \Delta$ as follows: $(\mathbb{Z} \Delta)_{0}=\mathbb{Z} \times \Delta_{0}$; the translation $\tau$ is given by $\tau((i, a))=(i-1, a)$, for $i \in \mathbb{Z}, a \in \Delta_{0}$; there are arrows $(i, a) \rightarrow(i, b)$ and $(i, b) \rightarrow(i+1, a)$ for any arrow $a \rightarrow b$ in $\Delta$ and any $i \in \mathbb{Z}$, and the valuation for these arrow is

$$
\begin{aligned}
& d_{(i, a)(i, b)}=d_{a b}=d_{(i, b)(i+1, a)}^{\prime}, \\
& d_{(i, a)(i, b)}^{\prime}=d_{a b}^{\prime}=d_{(i, b)(i+1, a)}^{\prime} .
\end{aligned}
$$

Since our translation quivers are allowed to have loops (but not multiple arrows), the notion of a covering as defined by Riedtmann [9] has to be modified: let $\left(\Gamma_{0}, \Gamma_{1}, \tau\right)$ and $\left(\Gamma_{0}^{\prime}, \Gamma_{1}^{\prime}, \tau\right)$ be stable translation quivers (without multiple arrows); a map

$$
\pi:\left(\Gamma_{0}, \Gamma_{1}, \tau\right) \rightarrow\left(\Gamma_{0}^{\prime}, \Gamma_{1}^{\prime}, \tau\right)
$$

is of the form $\pi=\left(\pi_{0}, \pi_{1}\right)$, where $\pi_{0}: \Gamma_{0} \rightarrow \Gamma_{0}^{\prime}, \pi_{1}: \Gamma_{1} \rightarrow \Gamma_{1}^{\prime}$ are set-theoretical maps such that for $\alpha: x \rightarrow y$, we have $\pi_{1}(\alpha): \pi_{0}(x) \rightarrow \pi_{0}(y)$, and $\pi_{0}(\tau(x))=\tau\left(\pi_{0}(x)\right.$ ), for all non-projective $x$. Such a map is called a covering provided $\pi_{0}$ and $\pi_{1}$ are surjective, and the restriction of $\pi_{0}$ to $x^{+}$is a bijection from $x^{+}$onto $\pi_{0}(x)^{+}$, and its restriction to $x^{-}$is a bijection from $x^{-}$onto $\pi_{0}(x)^{-}$. The covering $\pi$ is said to be finite, provided $\pi_{0}^{-1}(x)$ is finite, for every vertex $x$ of $\Gamma^{\prime}$. If $\Gamma, \Gamma^{\prime}$ are valued translation quivers, a map $\pi: \Gamma \rightarrow \Gamma^{\prime}$ is given by a map $\pi:\left(\Gamma_{0}, \Gamma_{1}, \tau\right) \rightarrow\left(\Gamma_{0}^{\prime}, \Gamma_{1}^{\prime}, \tau\right)$ such that $d_{\pi(x) \pi(y)}=d_{x y}, d_{\pi(x) \pi(y)}^{\prime}=d_{x y}^{\prime}$, for any arrow $x \rightarrow y$. A covering of valued translation quivers is a map of valued translation quivers which is a covering of translation quivers.

Proposition. Assume there exists a covering $\pi: \mathbb{Z} \Delta \rightarrow \Gamma(\mathscr{C})$, where $\Delta$ is either a finite valued quiver without oriented cycles or else $\Delta=\mathbb{A}_{\infty}^{\infty}, \mathbb{B}_{\infty}, \mathbb{C}_{\infty}$, or $\mathbb{D}_{\infty}$. Then, for every $l \in \mathbb{N}$, there are only finitely many isomorphism classes of indecomposable modules in $\mathscr{C}$ which have length $l($ in $\mathscr{A})$. Also, $\pi$ is a finite covering.

Proof. On $\mathbb{Z} \Delta$, we define an additive function $f$ by $f(x)=|\pi(x)|$. We show that for every $l \in \mathbb{N}$, the set $f^{-1}(l)=\left\{x \in(\mathbb{Z} \Delta)_{0} \mid f(x)=l\right\}$ is finite. This obviously yields both assertions. Assume $f^{-1}(l)$ is an infinite set and let us derive a contradiction.

First, assume $\Delta$ is a finite valued quiver (without oriented cycles). Since $\mathbb{Z} A$ has only finitely many $\tau$-orbits, some $\tau$-orbit contains infinitely many vertices from $f^{-1}(l)$. Thus, there is $a \in \Delta_{0}$ and an infinite subset $I \subseteq \mathbb{Z}$, such that $f((i, a))=l$, for all $i \in I$. We can assume that $a$ is the unique sink of $\Delta$. Let $\mathscr{C}(i)$ be the additive subcategory generated by all indecomposable objects $X$ in $\mathscr{C}$, with $[X]=\pi((i, b))$ for some $b \in A_{0}$. If $Z$ is indecomposable in $\mathscr{C}$, then $\left|\tau_{\mathscr{K}} Z\right| \leqq d|Z|$; thus, if $Y \rightarrow Z$ is an irreducible map of $\mathscr{K}$, then $|Y| \leqq(d+1)|Z|$. Since $\Delta$ has no oriented cycles, there is a bound $n$ on the length of all paths in $\Delta$. Given $b \in A_{0}$, there is a path from $b$ to $a$ in $\Delta$ of length $\leqq n$. Thus, for $i \in \mathbb{Z}$, there is a path in $\Gamma(\mathscr{C})$ from $\pi(i, b))$ to $\pi((i, a))$ of length $\leqq n$. It follows that for $i \in I$,

$$
|\pi((b, i))| \leqq(d+1)^{n}|\pi((a, i))|=(d+1)^{n} l .
$$

Let $l^{\prime}=(d+1)^{n} l$. Then $|Z| \leqq l^{\prime}$ for any indecomposable object $Z$ in $\mathscr{C}(i)$ with $i \in I$. 
Let $X, X^{\prime}$ be indecomposable objects in $\mathscr{K} \backslash \mathscr{C}$, and let $C, C^{\prime}$ be indecomposable objects in $\mathscr{C}$, such that $\operatorname{Hom}(X, C) \neq 0, \operatorname{Hom}\left(C^{\prime}, X^{\prime}\right) \neq 0$. Let $[C]=\pi((j, c)),\left[C^{\prime}\right]=\pi\left(\left(j^{\prime}, c^{\prime}\right)\right)$. First, suppose there are infinitely many $i \in I$ satisfying $i \leqq j$. Let $0 \neq \varphi: X \rightarrow C$. Using the sink map of the indecomposable objects in $\mathscr{C}(j)$, we can factor $\varphi=\psi_{1} \varphi_{1}$, where $\psi_{1} \in \operatorname{Hom}(X, C), \varphi_{1} \in \operatorname{rad}\left(C_{1}, C\right)$, with $C_{1} \in \mathscr{C}(j-1)$. By induction, we obtain a factorization $\varphi=\psi_{m} \varphi_{m} \cdots \varphi_{1}$, where $\psi_{m} \in \operatorname{Hom}\left(X, C_{m}\right), \varphi_{i} \in \operatorname{rad}\left(\left(C_{i}, C_{i-1}\right)\right.$, with $C_{i} \in \mathscr{C}(j-i)$, and $C_{0}=C$. However, as soon as the intervall $[j-m, j]$ in $\mathbb{Z}$ contains $2^{l^{\prime}}$ elements from $I$, the Harada-Sai lemma $[6,13]$ asserts that $\psi_{m} \cdots \varphi_{1}=0$. This contradicts our assumption $\varphi \neq 0$, and shows that there are only finitely many $i \in I$ satisfying $i \leqq j$. Similarly, if we suppose that there are infinitely many $i \in I$ satisfying $i \geqq j^{\prime}$, we use source maps in order to factorize any $0 \neq \varphi^{\prime}: C^{\prime} \rightarrow X^{\prime}$ in the form $\varphi^{\prime}=\varphi_{1}^{\prime} \cdots \varphi_{m}^{\prime} \psi_{m}^{\prime}$, where $\varphi_{i}^{\prime} \in \operatorname{rad}\left(C_{i-1}^{\prime}, C_{i}^{\prime}\right), \psi_{m}^{\prime} \in \operatorname{Hom}\left(C_{m}^{\prime}, X^{\prime}\right)$, with $C_{0}^{\prime}=C^{\prime}$, and $C_{i}^{\prime} \in \mathscr{C}\left(j^{r}+i\right)$, and again the Harada-Sai lemma gives a contradiction. This shows that there are only finitely many $i \in I$ satisfying $i \geqq j^{\prime}$. Thus, $I$ is finite. This finishes the proof in the case that $\Delta$ is finite.

Before we proceed to study the remaining cases, let us insert the following lemma on sectional paths due to Bongartz [2] and Igusa-Todorov [8].

Lemma. Let $\mathscr{K}$ be a Krull-Schmidt category with short exact sequences, and having sink maps. Assume the sink map for any indecomposable object $Z$ with [Z] a projective vertex in $\Gamma(\mathscr{K})$, is a monomorphism in $\mathscr{K}$. Let $X_{0}, \ldots, X_{n}$ be indecomposable modules, and $f_{i}: X_{i \sim 1} \rightarrow X_{i}, 1 \leqq i \leqq n$, irreducible maps. Assume that for all $2 \leqq i \leqq n$, either $X_{i}$ is projective, or $\tau_{\mathscr{K}} X_{i} \supsetneqq X_{i-2}$. Then $f_{1} \cdots f_{n} \neq 0$.

Pro of. (For the convenience of the reader, we sketch a short proof). Assume we have $f_{1} \cdots f_{n}=0$. Since irreducible maps are non-zero, we have $n \geqq 2$. We can assume that the $X_{i}, f_{i}$ are chosen in such a way that $n$ is minimal. In particular, $\left[X_{n}\right]$ is not a projective vertex in $I(\mathscr{K})$, since otherwise $f_{1} \ldots f_{n-1}=0$. Since $f_{n}$ is irreducible, there exists an Auslander-Reiten sequence of the form

$$
0 \rightarrow \tau_{\mathscr{K}} X_{n} \stackrel{\left[g_{n} *\right]}{\longrightarrow} X_{n-1} \oplus X_{n-1}^{\prime} \stackrel{\left[\begin{array}{c}
f_{n} \\
*
\end{array}\right]}{\longrightarrow} X_{n} \rightarrow 0
$$

Assume for some $t \geqq 2$, the vertices $\left[x_{i}\right], t \leqq i \leqq n$, are non-projective in $\Gamma(\mathscr{K})$. Then, for $n>i \geqq t$, we obtain inductively Auslander-Reiten sequences

$$
0 \rightarrow \tau_{\mathscr{K}} X_{i} \stackrel{\left[g_{i * *]}\right.}{\longrightarrow} X_{i-1} \oplus \tau_{\mathscr{K}} X_{i+1} \oplus X_{i-1}^{\prime} \stackrel{\left[\begin{array}{c}
f_{i} \\
g_{i+1}
\end{array}\right]}{\longrightarrow} X_{i} \rightarrow 0
$$

Here, we use that $\tau_{\mathscr{K}} X_{i+1} \neq X_{i-1}$. Since $f_{1} \cdots f_{n}=0$, there exists $h_{n}: X_{0} \rightarrow \tau_{\mathscr{K}} X_{n}$ with $h_{n} g_{n}=f_{1} \cdots f_{n-1}$. Inductively, we obtain for $n>i \geqq t$ maps $h_{i}: X_{0} \rightarrow \tau_{\mathscr{K}} X_{i}$ with $h_{i} g_{i}=f_{1} \cdots f_{i-1}$. If $t=2$, then $h_{t} g_{t}=f_{1}$ is a factorization of the irreducible map $f_{1}$ with $g_{t}$ not a split epimorphism, thus, $h_{t}$ has to be a split monomorphism, thus an isomorphism, but this means $X_{0} \approx \tau_{\mathscr{K}} X_{2}$, impossible. Thus, $t \geqq 3$, and we can assume that $\left[X_{t-1}\right]$ is a projective vertex in $\Gamma(\mathscr{K})$. The sink map for $X_{t-1}$ is of the form

$$
X_{t-2} \oplus \tau_{\mathscr{K}} X_{t} \oplus X_{t-2}^{\prime} \stackrel{\left[\begin{array}{c}
f_{t-1} \\
g_{t} \\
*
\end{array}\right]}{\longrightarrow} X_{t-1},
$$


and by assumption, this is a monomorphism in $\mathscr{K}$. Now

$$
\left[f_{1} \cdots f_{t-2},-h_{t}, 0\right]\left[\begin{array}{c}
f t-1 \\
g_{t} \\
*
\end{array}\right]=0,
$$

thus $f_{1} \cdots f_{t-2}=0$. But this contradicts the minimality of $n$. This finishes the proof.

R e m a rk. This lemma gives a direct proof to a result due to Bautista and Smalø [4]: The Auslander-Reiten quiver of an artin algebra does not have sectional cyclic paths.

Next, consider the case $\Delta=\mathbb{A}_{\infty}^{\infty}$. We assume that $\mathbb{A}_{\infty}^{\infty}$ has a set of vertices the set $\mathbb{Z}$ of integers, and that there are arrows $a \rightarrow a+1$, for any $a \in \mathbb{Z}$. It follows that the set of vertices of $\mathbb{Z} \mathbb{A}_{\infty}^{\infty}$ is $\mathbb{Z} \times \mathbb{Z}$, with arrows $(i, a) \rightarrow(i, a+1)$ and $(i, a) \rightarrow(i+1, a-1)$, for all $i, a \in \mathbb{Z}$. Let $g$ be an additive function on $\mathbb{Z} \mathbb{A}_{\infty}^{\infty}$. Then, for all $i, j, a, b \in \mathbb{Z}$,

$$
g((i, a))+g((j, b))=g((i, b+j-i))+g((j, a+i-j)) .
$$

In particular

$$
g((i, a))+g((0,0))=g((i,-i))+g((0, a+i)),
$$

for all $i, a \in \mathbb{Z}$. Assume that $g$ takes values in $\mathbb{N}_{0}$, and let $l_{0}=g((0,0))$. If $g((i, a))=l$, then both

$$
g((i,-i)) \leqq l+l_{0} \text { and } g((0, a+i)) \leqq l+l_{0} .
$$

Assume there are infinitely many vertices $(i, a)$ with $g((i, a))=l$. If there exists some fixed $i_{0}$, and infinitely many different $a_{s}, s \in \mathbb{N}$, with $g\left(\left(i_{0}, a_{s}\right)\right)=l$ for all $s \in \mathbb{N}$, then there are infinitely many $b_{s}, s \in \mathbb{N}$, with $g\left(\left(0, b_{s}\right)\right) \leqq l+l_{0}$, namely all the $b_{s}=a_{s}+i_{0}$. Otherwise, there must exist infinitely many different $i_{s}, s \in \mathbb{N}$, and for any $i_{s}$ some $a_{s}^{\prime}$ with $g\left(\left(i_{s}, a_{s}^{\prime}\right)\right)=l$; in this case $g\left(\left(i_{s},-i_{s}\right)\right) \leqq l+l_{0}$. It follows that for some fixed value $l^{\prime}\left(\leqq l+l_{0}\right)$, there are infinitely many different vertices $(i, a)$ with $g((i, a))=l^{\prime}$ and $i=0$ or $i+a=0$.

We apply this to the given additive function $f(x)=|\pi(x)|$. We see that there is a fixed value $l^{\prime}$ and infinitely many different $a \in \mathbb{Z}$ such that either $f((0, a))=l^{\prime}$ or $f((-a, a))=l^{\prime}$. First, assume there are infinitely many different $a \in \mathbb{Z}$ such that $f((0, a))=l^{\prime}$. Take integers $u, v$ such that there are at least $2^{l^{\prime}}$ different $a \in \mathbb{Z}$ with $u \leqq a \leqq v$ and $f((0, a))=l^{\prime}$. For every $b \in \mathbb{Z}$, choose a representative $M(b) \in \pi((0, b))$, note that there exists an irreducible map $\alpha_{b}: M(b) \rightarrow M(b+1)$. The Harada-Sai lemma $[6,10]$ asserts that $\alpha_{u} \alpha_{u+1} \cdots \alpha_{v-1}=0$, whereas the lemma above yields that this composition is non-zero. In order to be able to apply our lemma, we have to observe that for all $i, a \in \mathbb{Z}$, we have $\pi((i-1, a)) \neq \pi((i, a-2))$, since $\pi$ is assumed to be a covering. Similarly, we proceed in the case that there are infinitely many $a \in \mathbb{Z}$ such that $f((-a, a))=l$. This finishes the proof for $\boldsymbol{A}=\mathbb{A}_{\infty}^{\infty}$.

Consider now $\Delta=\mathbb{B}_{\infty}, \mathbb{C}_{\infty}$, or $\mathbb{D}_{\infty}$. The set of vertices of $\Delta$ is $\mathbb{N}_{0}$ for $\Delta=\mathbb{B}_{\infty}, \mathbb{C}_{\infty}$, and $\mathbb{N}_{0} \cup\left\{0^{\prime}\right\}$ for $\Delta=\mathbb{D}_{\infty}$; there are arrows $a \rightarrow a+1$, for $a \in \mathbb{N}_{0}$, and, for $\Delta=\mathbb{D}_{\infty}$, an additional arrow $0^{\prime} \rightarrow 1$; the only nontrivial valuation is for the arrow $0 \rightarrow 1$ for $\Delta=\mathbb{B}_{\infty}$ and $\mathbb{C}_{\infty}$, namely $d_{01}=1, d_{01}^{\prime}=2$ for $\Delta=\mathbb{B}_{\infty}$, and $d_{01}=2, d_{01}^{\prime}=1$ for $\Delta=\mathbb{C}_{\infty}$. 
The function $f$ on $\mathbb{Z} \Delta$ defines a function $g$ on $\mathbb{Z} \mathbb{A}_{\infty}^{\infty}$ as follows: let

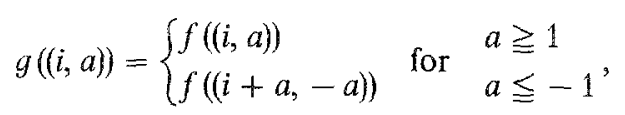

and

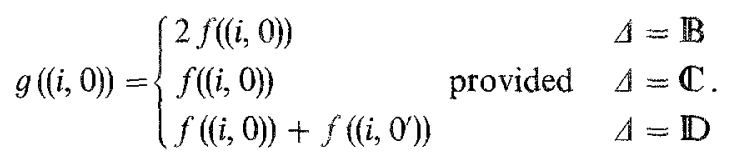

One easily checks that $g$ is additive on $\mathbb{Z} \mathbb{A}_{\infty}^{\infty}$, since $f$ is additive on $\mathbb{Z} \Delta$. It follows that for some fixed value $l^{\prime}$ there are infinitely many different vertices $(i, a) \in \mathbb{Z} \times \mathbb{Z}$ with $g((i, a))=l^{\prime}$ and $i=0$ or $i+a=0$. Consequently, there are infinitely many different $a \in \mathbb{N}_{1}$ such that either $f((0, a))=l^{\prime}$ or $f((-a, a))=l^{\prime}$. Again, we obtain a contradiction by using both the Harada-Sai lemma and our lemma above. This finishes the proof.

Rem a r $k$ 1. The special case of $\Delta$ being a star has been treated before in [1].

$\mathrm{R}$ e m a rk 2 . It is obvious that for $\Delta=\mathbb{A}_{\infty}^{\infty}, \mathbb{B}_{\infty}, \mathbb{C}_{\infty}, \mathbb{D}_{\infty}$, there are no finite coverings $\mathbb{Z} \Delta \rightarrow \Gamma$. This shows that under our assumptions, any component $\Gamma$ with a covering $\mathbb{Z} \Delta \rightarrow \Gamma, \Delta=\mathbb{A}_{\infty}^{\infty}, \mathbb{B}_{\infty}, \mathbb{C}_{\infty}, \mathbb{D}_{\infty}$ is actually of the form $\mathbb{Z} \Delta$. For $\mathscr{A}=A$-mod, this has been observed in [5] in the cases $\Delta=\mathbb{B}_{\infty}, \mathbb{C}_{\infty}, \mathbb{D}_{\infty}$, and in [3] in the case $\Delta=\mathbb{A}_{\infty}^{\infty}$.

\section{References}

[1] M. Auslander, R. Bautista, M. I. Platzeck, I. Reiten and S. O. Smalo, Almost split sequences whose middle term has at most two indecomposable summands. Canad. J. Math. 31, $942-960(1979)$.

[2] K. BongartZ, On a result of Bautista and Smalø on cycles. Comm. Algebra 11, 2123-2124 (1983).

[3] M. C. R. ButLer and C. M. RJngel, Auslander-Reiten sequences with few midale terms, with applications to string algebras. Comm. Algebra 15, 145-179 (1987).

[4] R. Bautista and S. O. Smalø, Nonexistent cycles. Comm. Algebra 11, 1755-1767 (1983).

[5] D. HapPeL, U. PreISER and C. M. RINGEL, Vinberg's characterization of Dynkin diagrams using subadditive functions with, application to DTr-periodic modules. LNM 832, 280-294, Berlin-Heidelberg-New York 1980.

[6] M. HARADA and Y. SAI, On categories of indecomposable modules I. Osaka J. Math. 7, $323-344(1970)$.

[7] M. Hoshino, Splitting torsion theories induced by tilting modules. Comm. Algebra 11, 427441 (1983).

[8] K. IGUSA and G. ToDorov, A characterization of finite Auslander-Reiten quivers. J. Algebra 89, $148-177$ (1984).

[9] Chr. RiedtMann, Algebren, Darstellungsköcher, Überlagerungen und zurück. Comment. Math. Helv. 55, 199-224 (1980).

[10] C. M. RINGeL, Report on the Brauer-Thrall conjectures. LNM 831, 104-136, Berlin-Heidelberg-New York 1980.

[11] C. M. RINGEL, Tame algebras and integral quadratic forms. LNM 1099, Berlin-HeidelbergNew York 1984. 
[12] C. M. RingeL, Representation theory of finite dimensional algebras. Durham lectures. In: Representations of Algebras. London Math. Soc. Lecture Note Series. 116, 7-79 (1986).

[13] C. M. RINGEL, The regular components of the Auslander-Reiten quiver of a tilted algebra. To appear in Chinese Ann. Math.

Eingegangen am 22.11.1986

Anschrift der Autoren:

Eugenia Marmolejo

Claus Michael Ringel

Fakultät für Mathematik

Universität Bielefeld

Universitätsstr. 1

D-4800 Bielefeld 1 